\title{
Effects of tumor size on prognosis in differentiated thyroid carcinoma smaller than $2 \mathrm{~cm}$
}

\author{
TING-TING ZHANG ${ }^{1}$, CUN-FU LI ${ }^{2}$, SHI-SHUAI WEN ${ }^{1}$, DONG-ZHE HUANG ${ }^{1}$, GUO-HUA SUN ${ }^{1}$, \\ YONG-XUE ZHU ${ }^{1}$, YU WANG ${ }^{1}$, QING-HAI JI ${ }^{1}$, NING QU ${ }^{1}$ and RONG-LIANG SHI ${ }^{1}$ \\ ${ }^{1}$ Department of Head and Neck Surgery, Fudan University Shanghai Cancer Center, Shanghai 200032; \\ ${ }^{2}$ Department of General Surgery, Weihai Central Hospital, Weihai, Shandong 264400, P.R. China
}

Received September 28, 2018; Accepted October 9, 2018

DOI: $10.3892 / 01.2019 .10088$

\begin{abstract}
Benefits of subdividing small-differentiated thyroid carcinoma (sDTC) by tumor size are controversial. We conducted a meta-analysis to investigate whether tumor size is associated with prognosis of sDTC. PubMed and Web of Science databases were searched from their inception to September 2018. The identified studies according to the inclusion/exclusion criteria were analyzed using fixed/random-effects models. Data were calculated and results of the meta-analysis were expressed as odd ratio (OR). sDTC was classified as $S 1(\leq 1 \mathrm{~cm})$ and $S 2(>1 \mathrm{~cm}$ and $\leq 2 \mathrm{~cm}$ ). A systematic analysis was performed to compare the difference of recurrence, survival and clinicopathological factors between the two subgroups of sDTC (S1 vs. S2). A total of 21 studies published between 2004 and 2017 enrolling 219,291 patients were included. Findings showed that, $S 2$ was associated with higher recurrence risk compared with S1 $(\mathrm{OR}=1.575,95 \% \mathrm{CI}=1.428-1.738 ; \mathrm{P}<0.05)$. There was no statistical difference in survival between $S 1$ and $S 2$, but significant statistical heterogeneity $(\mathrm{OR}=1.160,95 \% \mathrm{CI}=0.810-1.662$; $\left.\mathrm{P}=0.448 ; \mathrm{I}^{2}=75.8 \%\right)$. Meta-regression analysis revealed publication year potentially caused the heterogeneity $(\mathrm{P}<0.05)$. Comparison of small papillary thyroid carcinoma alone agreed with the results of sDTC. T1b increased the risk of recurrence $(\mathrm{OR}=1.520 ; 95 \% \mathrm{CI}=1.072-2.155 ; \mathrm{P}<0.05)$ and death $(\mathrm{OR}=1.504$; 95\% CI 1.353-1.672; $\mathrm{P}<0.05)$ compared with T1a. S2 associated with extrathyroidal extension $(\mathrm{OR}=2.575 ; 95 \% \mathrm{CI}=1.603-4.135$; $\mathrm{P}<0.05)$, bilaterality $(\mathrm{OR}=2.278 ; 95 \% \mathrm{CI}=1.905-2.723 ; \mathrm{P}<0.05)$, vascular invasion $(\mathrm{OR}=4.494 ; 95 \% \mathrm{CI}=2.812-7.183 ; \mathrm{P}<0.05)$ and lymph node metastases ( $\mathrm{OR}=1.12$; 95\% CI=1.10-1.14; $\mathrm{P}<0.05)$. Our analysis suggested it is necessary to subdivide sDTC into S1
\end{abstract}

Correspondence to: Professor Rong-Liang Shi or Professor Ning Qu, Department of Head and Neck Surgery, Fudan University Shanghai Cancer Center, 270 Dongan Road, Shanghai 200032, P.R. China

E-mail: shirongliang@126.com

E-mail: jonathan_qn@163.com

Key words: differentiated thyroid carcinoma, tumor size, recurrence, prognosis and S2 owing to their different effects on prognosis, especially recurrence.

\section{Introduction}

Differentiated thyroid carcinoma (DTC), which mainly includes cancers of papillary and follicular histologies, is the most common form of thyroid cancer (1) DTC typically has a favorable prognosis, with an overall 10-year survival rate above $90 \%$. As reported by epidemiological studies, the incidence of thyroid cancer has been on the increase in previous decades (2). The vast majority $(87 \%)$ of thyroid cancers detected in the last 15 years were diagnosed as small DTC (sDTC), which is defined as tumors $\leq 2 \mathrm{~cm}$ in their largest diameter $(3,4)$. Thus, defining the appropriate treatment and management strategies for patients with early stage DTC, especially sDTC, is necessary.

In the 6th edition of the Union for International Cancer Control (UICC)/American Joint Committee on Cancer (AJCC) (5-7), the tumor, node, metastases (TNM) staging system defined intrathyroidal tumors, with the largest diameter being $\leq 2 \mathrm{~cm}$, as T1. Previous findings have suggested that tumors $>1 \mathrm{~cm}$ have a worse prognosis (8). Thus, the 7 th AJCC TNM staging system made a subdivision of T1 tumors into T1a $(\leq 1 \mathrm{~cm})$ and T1b $(1-2 \mathrm{~cm})(9)$. Furthermore, the 8th AJCC TNM staging system continued to use T1a/T1b (10). The 2015 American Thyroid Association guidelines recommended different therapeutic management for the two DTC subgroups (11). However, in other studies it was emphasized that smaller DTCs did not indicate better prognosis, and that there was no difference between the two subgroups (12-14). Due to the inconsistent conclusions of the previous respective studies and a lack of prospective studies, whether it is clinically beneficial to subdivide sDTCs remains controversial.

The aim of the present study was to investigate whether the subgroups (group $S 1$, defined as tumors $\leq 1 \mathrm{~cm}$ and group $S 2$, defined as tumors $>1 \mathrm{~cm}$ and $\leq 2 \mathrm{~cm}$ ) are distinguishable based on patient prognosis.

\section{Materials and methods}

Search strategy and study selection. PRISMA-P (Preferred Reporting Items for Systematic review and Meta-Analysis Protocols) was referred in the process of our 
meta-analysis (15). A comprehensive literature search for studies published before September 2018 was performed in the PubMed and Web of Science databases. We used the following keywords as the search algorithm: ('differentiated thyroid carcinoma' OR 'DTC') AND ('follow up' OR 'result' OR 'prognosis' OR 'death' OR 'recurrence') AND ('tumor size' OR 'T1') AND 'patients'. All of the reference lists from the main articles were inspected for additional eligible studies.

Studies were considered eligible if they met the following inclusion criteria: i) The original publication was in English; ii) all enrolled patients received surgery without other forms of treatments (including radiotherapy, chemotherapy and targeted therapy); iii) studies were about primary tumors; iv) baseline characteristics of enrolled patients were described in detail (including sex, age, and treatment); v) studies provided information regarding recurrence and death events in relation to clinicopathological factors of the patients.

First, abstracts of all identified citations were screened, and those not meeting the inclusion criteria were excluded. Subsequently, the full texts of the remaining articles were rescreened, and studies were excluded if they met the following exclusion criteria: i) Small sample size $(\leq 20)$; ii) studies lacking prognosis or recurrence data classified by tumor size; iii) articles that only offered the relative information of one group; iv) tumor sizes were measured using imaging data, instead of in surgery or pathological specimen.

Data extraction and quality assessment. According to the inclusion/exclusion criteria, 21 studies were included in the current meta-analysis. We extracted the following data from each study: the first author's name, year of publication, study location, number of patients included, study design, period of follow-up, sex composition, therapeutic method, and odds ratios (ORs) with their 95\% confidence intervals (95\% CIs), resulting from univariate analysis (either published or derived from reported data).

Study quality was scored using the Newcastle-Ottawa Scale (NOS) (16). The NOS is frequently used for non-random studies, such as cohort studies. The maximum score for a cohort study was 9 , and studies scoring between 5 and 9 are generally considered high quality. The quality scores of the 21 studies ranged from 7 to 9 , and all were considered adequate for inclusion in the meta-analysis.

Ethics approval. The present study was approved by the Human Ethics Committee/Institutional Review Board of Fudan University Shanghai Cancer Center (Shanghai, China).

Statistical analysis. The meta-analysis was performed using STATA version 12.0 program (StataCorp, College Station, TX, USA) to facilitate the pooling of results across studies. The final results are expressed as an OR (odds ratio) and its 95\% confidence interval (CI). Heterogeneity in each study was assessed using $\chi^{2}$ tests (Q-value and $\mathrm{P}$-value) and $\mathrm{I}^{2}$ measures. Significant heterogeneity was defined as a $\chi^{2}$ test with $\mathrm{P}<0.05$ or $\mathrm{I}^{2}>50 \%$. Random-effect models (Mantel-Haenszel and Der Simonnian-Laird methods) were then used for primary analyses in datasets with significant heterogeneity. Primary datasets without significant heterogeneity were analyzed using fixed-effect models (Mantel-Haenszel method). If there was significant heterogeneity and $>10$ studies included, we accounted for statistical heterogeneity by meta-regression analysis. Publication biases were assessed by Begg's test in each meta-analysis, and we assumed publication bias was present if $\mathrm{P}<0.05$.

\section{Results}

Baseline study and patient characteristics. The process of selecting studies according to the inclusion/exclusion criteria is shown in Fig. 1, whereby 21 studies were included in the final meta-analysis (5-8, 12-14, 17-30). The principal characteristics of the 21 studies are shown in Table I. In total 219,291 patients were involved, and the mean age of the enrolled patients in each study was similar. All 21 studies were retrospective cohort studies published between 2004 and 2017, except one prospective study. The follow-up time ranged from 3.6 to 14.1 years. The main treatment was total thyroidectomy (TT) or near total thyroidectomy (nTT) with postoperative radioiodine as selective adjuvant therapy.

All the NOS scores of the eligible studies were greater than 5 for the 9 questions, with an average of 8.05 (range, 7-9), indicating good quality for meta-analysis. The included studies were all cohort studies with definitive controls for selection, yielding the good scores.

Association between tumor size and recurrence in DTC. As shown in Fig. 2A, the risk of recurrence for DTC patients based on tumor size was compared in 10 studies $(6,8,12-14,17$, $21,23,26-27)$. In the meta-analysis, larger tumor size ( $S 2$ vs. $S 1$ ) was associated with higher risk of postoperative recurrence in sDTC patients $(\mathrm{OR}=1.575 ; 95 \% \mathrm{CI}=1.428-1.738$; $\mathrm{P}<0.05)$. No significant statistical heterogeneity was detected among these studies $\left(\mathrm{P}=0.653 ; \mathrm{I}^{2}=0.0 \%\right)$.

The prognostic influence of tumor size is partly attributable to its association with more aggressive histologic features such as extrathyroidal extension (ETE), rather than the impact of size itself. Thus, our analysis compared the influence of T1a and T1b on recurrence based on the data from four studies $(12-14,17)$. In the meta-analysis, T1b also indicated higher risk of recurrence than T1a $(\mathrm{OR}=1.520$; 95\% CI=1.072-2.155; P<0.05) (Fig. 2B). There was no significant statistical heterogeneity among these studies $\left(\mathrm{P}=0.168, \mathrm{I}^{2}=40.6 \%\right)$.

Association between tumor size and survival in DTC. Eleven articles provided survival data for groups $S 1$ and $S 2(5-8,12,17,18,21,23,25,27)$. Due to the favorable prognosis of DTC, there was no end-point mortality data in 5 studies. Based on the random-effect meta-analysis, tumor size (S1 vs. S2) had no association with survival in DTC patients $(\mathrm{OR}=1.160,95 \% \mathrm{CI}=0.810-1.662 ; \mathrm{P}=0.448)$ (Fig. 3A). However, significant statistical heterogeneity was detected among these studies $\left(\mathrm{P}<0.05, \mathrm{I}^{2}=75.8 \%\right)$. Thus, a meta-regression analysis was performed to explore the sources of heterogeneity. Study location $(\mathrm{P}=0.49)$, cohort size $(\mathrm{P}=0.286)$, follow-up period $(\mathrm{P}=0.282)$, patient age $(\mathrm{P}=0.131)$ and sex composition $(\mathrm{P}=0.866)$ were not the sources of heterogeneity. However, the year of publication potentially caused statistical heterogeneity $(\mathrm{P}<0.05)$. Subgroup analysis of the nine studies published after 


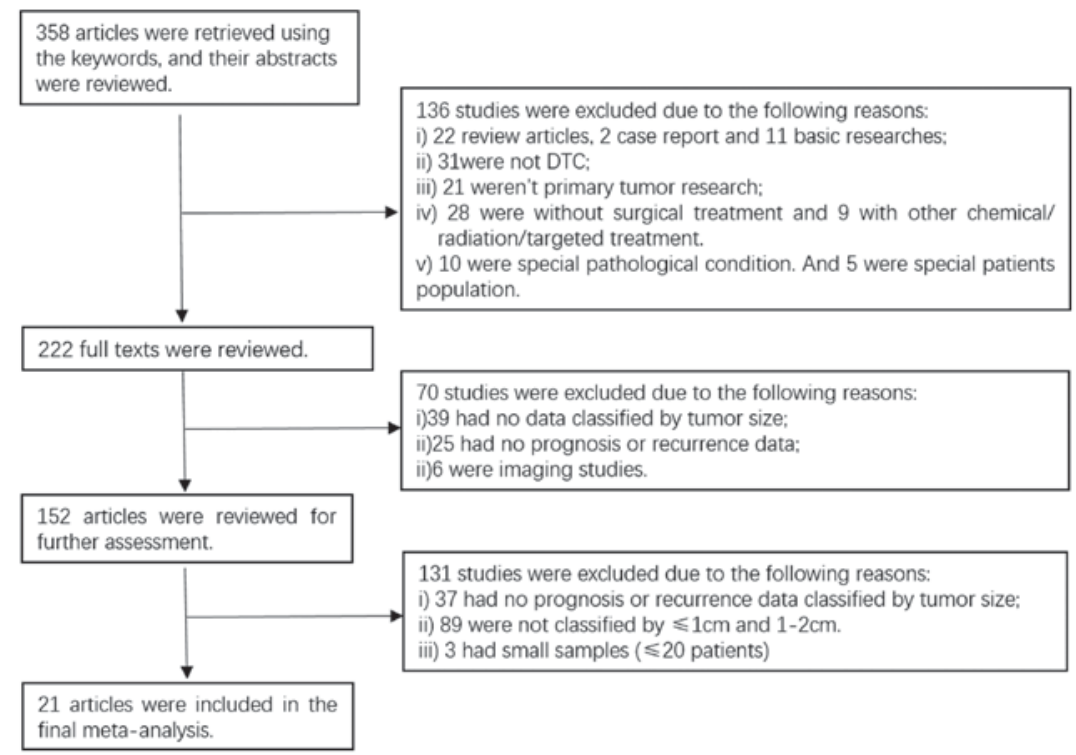

Figure 1. Process of searching and screening for articles according to the inclusion/exclusion criteria.

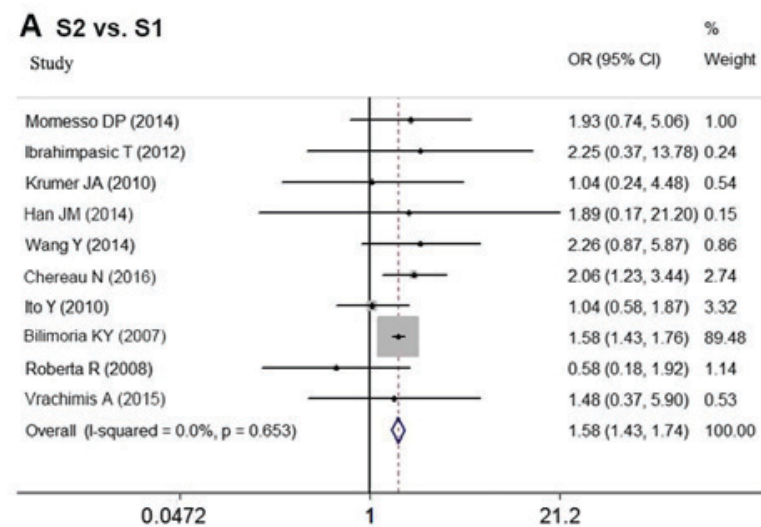

B T1b vs. T1a

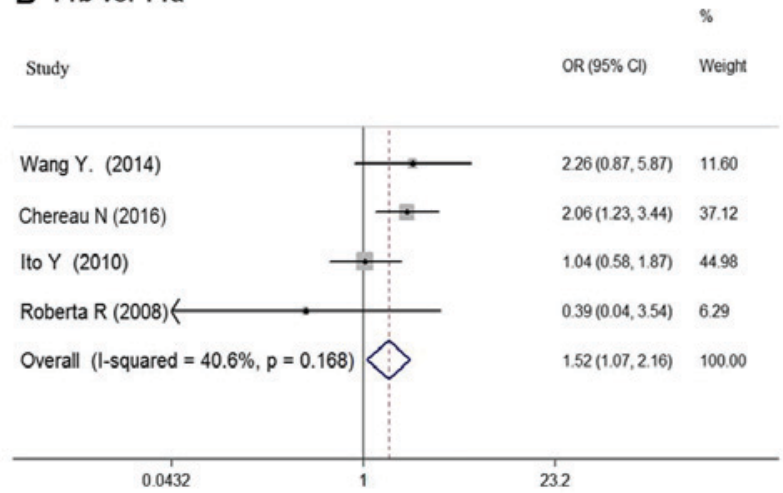

Figure 2. The fixed effects model of the OR for the association between tumor size and recurrence in sDTC is shown with 95\% CIs. (A) Comparison between groups S2 vs. S1, (B) Comparison between T1b and T1a. CI, confidence interval; OR, odds ratio; sDTC, small-differentiated thyroid carcinoma.

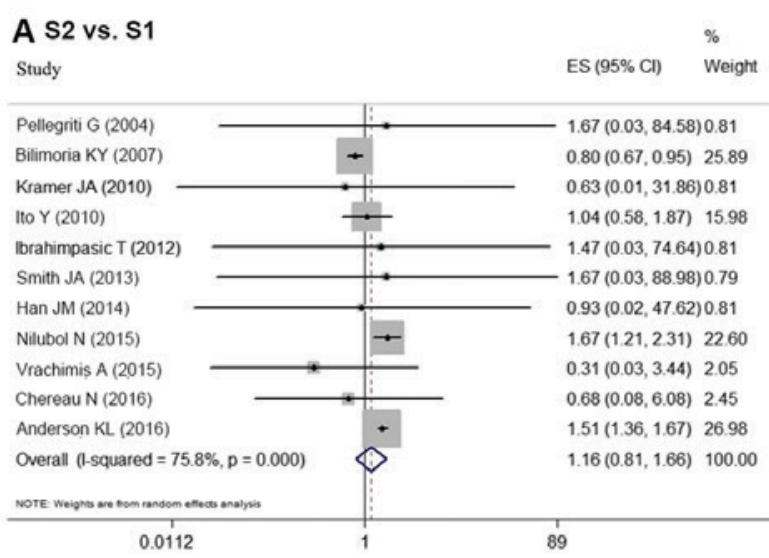

\section{B T1b vs. T1a}

Study

ES $(95 \% \mathrm{Cl}) \quad$ Weight

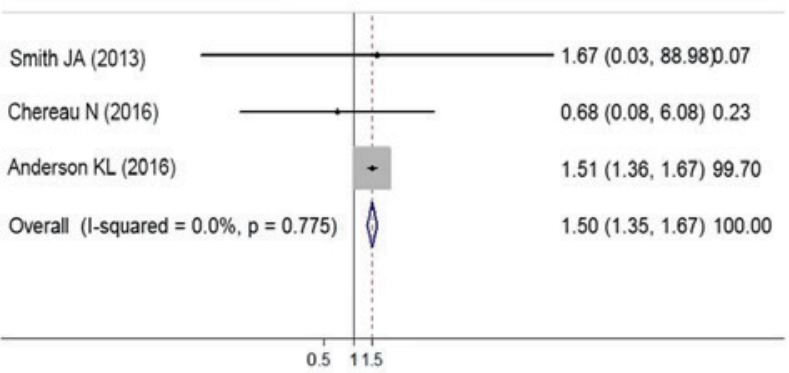

Figure 3. The OR for the association between tumor size and survival in SDTC is shown with 95\% CIs. (A) Comparison between group S2 vs. S1 using the random effects model, and (B) Tlb and Tla using the fixed effects model. CI, confidence interval; OR, odds ratio; sDTC, small-differentiated thyroid carcinoma.

$2009(5-7,12,17,21,23,25,27)$ indicated group $S 2$ had a worse prognosis compared with $S 1(\mathrm{OR}=1.498,95 \% \mathrm{CI}=1.357-1.653$; $\mathrm{P}<0.05)$. However, an analysis of the two studies conducted prior to 2009 showed the opposite conclusion $(\mathrm{OR}=0.800$; 95\% CI=0.670-0.955; P<0.05) $(8,18)$ (Table II; Fig. 4). There was no significant statistical heterogeneity among the studies 


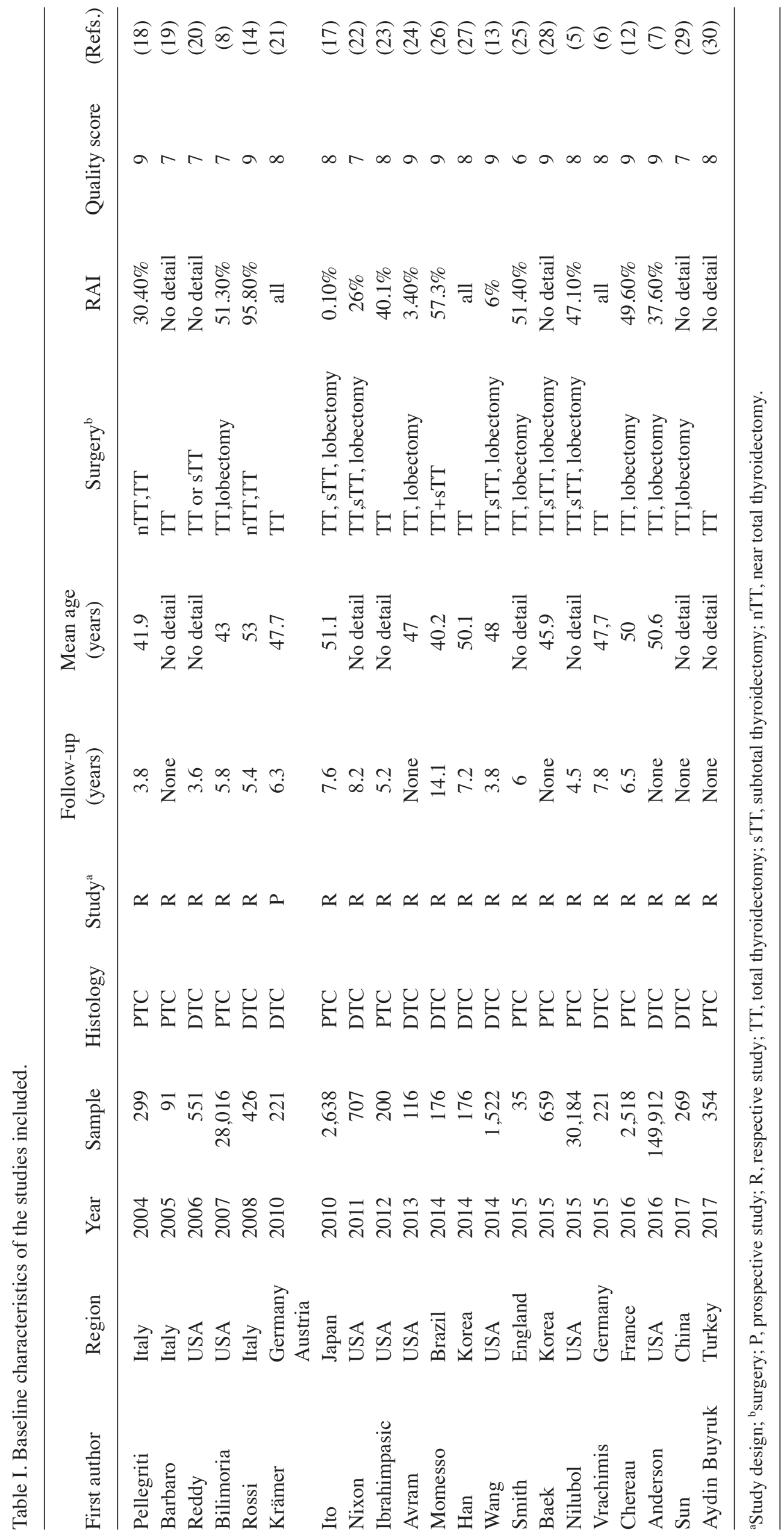


Table II. Subgroup analysis of association between tumor size and prognosis.

\begin{tabular}{llccccrr}
\hline Factor & Standard & No. & Q-value & P-value & $I^{2}$ & OR & 95\% CI \\
\hline Year & Before 2009 & 2 & 0.13 & 0.014 & $0.0 \%$ & 0.800 \\
& After 2009 & 9 & 4.33 & 0.000 & $0.0 \%$ & 1.498 & $1.357-1.653$ \\
\hline
\end{tabular}

No., the number of articles included; CI, confidence interval; OR, odds ratio.

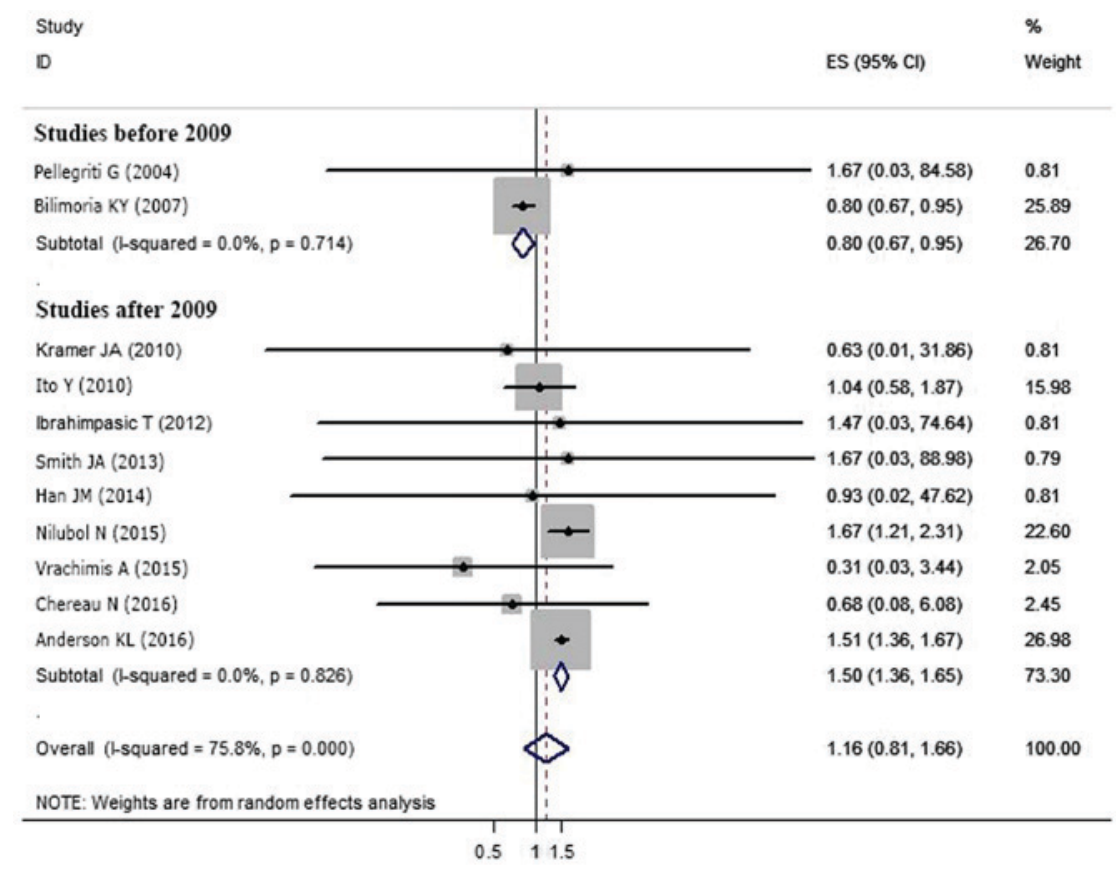

Figure 4. Subgroup meta-analysis for the difference in the impact on survival between S1 and S2 in sDTC classified by the year of publication is shown as the odds ratio with $95 \%$ CIs. CI, confidence interval; sDTC, small-differentiated thyroid carcinoma.

when divided by publication date (before 2009, $\mathrm{P}=0.714$, $\mathrm{I}^{2}=0.0 \%$; after 2009, $\mathrm{P}=0.826, \mathrm{I}^{2}=0.0 \%$ ).

Aggressive histological features can also affect prognosis; therefore, $\mathrm{T}$ stage was analyzed independently to determine whether T1b impacted postoperative survival compared with T1a. The meta-analysis of the data from three studies $(7,12,25)$ revealed that $\mathrm{T} 1 \mathrm{~b}$ indicated a worse prognosis compared with T1a $(\mathrm{OR}=1.504,95 \% \mathrm{CI}=1.353-1.672 ; \mathrm{P}<0.05)$ (Fig. 3B). No statistical heterogeneity $\left(\mathrm{P}=0.775, \mathrm{I}^{2}=0.0 \%\right)$ was detected.

Association between tumor size and prognosis in PTC. Considering that different histologies (papillary thyroid, follicular thyroid and Hürthle cell cancer) indicate different prognoses, we independently analyzed the influence of the two groups ( $S 1$ vs. $S 2$ ) on the prognosis of PTC patients. The meta-analysis based on four studies $(8,12,17,23)$ revealed that larger tumor size $(S 2)$ increased the risk of recurrence for sPTC patients compared with $S 1(\mathrm{OR}=1.580,95 \% \mathrm{CI}=1.430-1.747$; $\mathrm{P}<0.05)$, and there was no statistical heterogeneity between these studies $\left(\mathrm{P}=0.337, \mathrm{I}^{2}=3.0 \%\right)$ (Fig. 5A). Due to the significant heterogeneity $\left(\mathrm{P}=0.015, \mathrm{I}^{2}=62.1 \%\right)$, the random effect model was used for the remaining seven studies $(5,8,12,17,18,23,25)$ to explore the subgroup effect on survival. The analysis revealed that there was no statistical difference between the two groups
$(S 2$ vs. $S 1)$ in survival $(\mathrm{OR}=1.101,95 \% \mathrm{CI}=0.708-1.712$; $\mathrm{P}=0.668)$ (Fig. 5B).

Tumor size and aggressive histologic features in DTC. The relationship between tumor size and aggressive histological features was also analyzed. Multifocality, ETE, bilaterality, vascular invasion, lymph node metastases and distant metastases were compared in eight $(12-14,18,19,24,25,28)$, five $(14,18,19,22,27)$, three $(12,18,28)$, four $(7,12,13,18)$, eleven $(6,7,12,14,18-20,24,28-30)$ and five $(7,12,14,18,24)$ studies, respectively. Based on the meta-analysis, $S 2$ was found to be associated with aggressive histological features more often compared with S1, including ETE (OR=2.575, 95\% $\mathrm{CI}=1.603-4.135 ; \mathrm{P}<0.05)$ (Fig. 6A), bilaterality $(\mathrm{OR}=2.278,95 \% \mathrm{CI}=1.905-2.723 ; \mathrm{P}<0.05)$ (Fig. 6B), vascular invasion $(\mathrm{OR}=4.494,95 \% \mathrm{CI}=2.812-7.183, \mathrm{P}<0.05)$ (Fig. 6C), lymph node metastases $(\mathrm{OR}=1.12,95 \% \mathrm{CI}=1.10-1.14$, $\mathrm{P}<0.05)$ (Fig. 6E) and distant metastases $(\mathrm{OR}=1.373$, 95\% CI=1.155-1.631; $\mathrm{P}<0.05$ ) (Fig. 6D). Although it has been reported that tumors $>1 \mathrm{~cm}$ are more frequently associated with multifocality than tumors $\leq 1 \mathrm{~cm}$, no significant association was found in our meta-analysis $(\mathrm{OR}=1.242,95 \% \mathrm{CI}$ 0.899-1.716; $\mathrm{P}=0.188$ ) (Fig. 6F). There was no statistical heterogeneity among these studies when evaluating the 

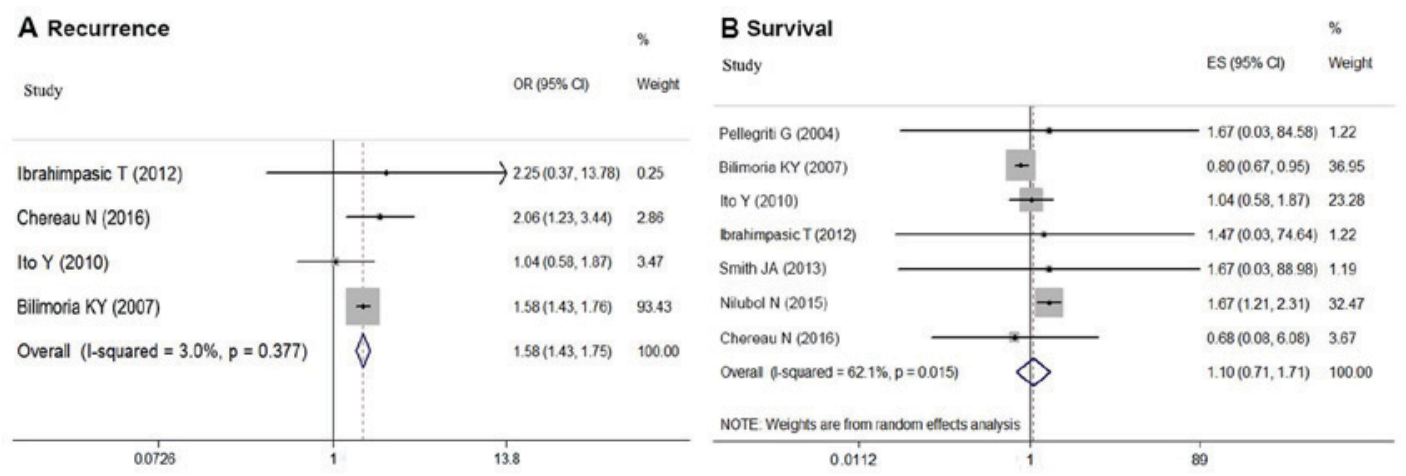

Figure 5. Meta-analysis results regarding the association between subgroups (S2 vs. S1) and prognosis in sPTC are shown as the ORs with 95\% CIs. (A) The fixed effects model comparing recurrence between subgroups. (B) The random effects model comparing survival between subgroups. CI, confidence interval; OR, odds ratio; sDTC, small-differentiated thyroid carcinoma.

association with bilaterality $\left(\mathrm{P}=0.632, I^{2}=0.0 \%\right)$, lymph node metastases $\left(\mathrm{P}=0.146, \mathrm{I}^{2}=31.6 \%\right)$, and distant metastases $\left(\mathrm{P}=0.717, I^{2}=0.0 \%\right)$. However, significant statistical heterogeneity was detected among the studies predicting correlations between tumor size and multifocality $\left(\mathrm{P}<0.05, \mathrm{I}^{2}=80.9 \%\right)$, extrathyroidal extension $\left(\mathrm{P}<0.05, \mathrm{I}^{2}=75.6 \%\right)$ and vascular invasion $\left(\mathrm{P}<0.05, \mathrm{I}^{2}=75.3 \%\right)$. Furthermore, Begg's test was negative for all analyses.

\section{Discussion}

Primary tumor diameter has been described as a determinant for outcome in DTC (9). The subdivision of sDTC is based on previous studies that indicated tumors $>1 \mathrm{~cm}$ had worse prognoses (8). However, it remains controversial whether the subgroups ( $S 1$ vs. $S 2$ ) influence the prognosis of sDTC and what the appropriate therapeutic strategies are for patients in each subgroup. Thus, we determined whether tumor size $>1 \mathrm{~cm}$ would impact recurrence and survival in SDTC. The results of our study offer some guidelines for physicians dealing with sDTC patients.

The current meta-analysis focused on the effects of tumor size ( $S 2$ vs. $S 1)$ on postoperative recurrence and survival. The results indicated that patients with $S 2$ had a higher risk of postoperative recurrence compared with $S 1$ during follow-up. However, there was no statistical difference in survival between patients within the groups, and significant statistical heterogeneity was detected during analysis. Based on meta-regression analysis, publication year potentially caused the statistical heterogeneity $(\mathrm{P}<0.05)$. In the subgroup meta-analysis, the subgroup including nine studies published after 2009 showed that $S 2$ was associated with worse prognoses compared with S1, but the subgroup of two studies published before 2009 showed the opposite; there were no statistical heterogeneities in either subgroup. Of note, the 7th AJCC's TNM staging guide was published in 2009, which subdivided T1 to T1a and $\mathrm{T} 1 \mathrm{~b}$ (9). This subdivision may heighten differences between the two subgroups. Further prospective studies are necessary to confirm the influence of tumor size on survival in SDTC.

To remove the effects of aggressive histological features such as ETE, we analyzed the influence of T stage (T1a vs. T1b) independently on postoperative prognosis. The results also showed that $\mathrm{T} 1 \mathrm{~b}$ increased the risk of recurrence and death compared with T1a. Since different pathological patterns of DTC indicate different prognoses, we analyzed the two groups (S1 vs. S2) in PTC independently. The meta-analysis revealed that $S 2$ increased the risk of recurrence for sPTC patients compared with $S 1$, but there was no statistical difference between the two groups on survival. Given that the prognostic influence of tumor size is partly due to its association with aggressive histological features, our study explored the correlation between tumor size and other clinicopathological factors. The results confirmed that $S 2 \mathrm{sDTC}$ was more often associated with ETE, bilaterality, vascular invasion, lymph node metastases and distant metastases than $S 1$.

The optimal treatment for the patients with sDTC is controversial. Bilimoria et al (8) reported a retrospective study enrolling 52,173 papillary thyroid carcinoma patients (including 28,016 sPTC patients) that concluded lobectomy as initial treatment was inadequate and had a worse prognosis regarding recurrence and death events compared with total thyroidectomy for the patients with $S 2$ disease (for recurrence: $\mathrm{HR}=1.24,95 \% \mathrm{CI}=1.01-1.65, \mathrm{P}=0.04$; for survival: $\mathrm{HR}=1.49$, 95\% CI=1.02-2.17, $\mathrm{P}=0.04$ ), but for patients with $S 1$ disease, the two surgical approaches made no difference in prognosis. However, the 2015 ATA guidelines also recommended that thyroid lobectomy alone may be sufficient initial treatment for low-risk DTC $\leq 4 \mathrm{~cm}$, unless there are clear indications to remove the contralateral lobe (11) Momesso et al (26) retrospectively analyzed clinical data from 176 DTC patients and argued that the surgical approach (total thyroidectomy or subtotal thyroidectomy) did not influence postoperative survival or recurrence for patients in either group; they also found no influence from radioiodine therapy. Ito et al (17) investigated the prognosis of 2,638 patients with T1N0M0 papillary thyroid carcinoma (PTC) who underwent initial surgery without radioiodine therapy. Those authors concluded that total thyroidectomy is not mandatory for T1N0M0 PTC patients unless other diseases coexisted requiring total thyroidectomy if a $1 \%$ risk of recurrence to the remnant thyroid is acceptable, and radioiodine ablation therapy is also not necessary. Based on a retrospective study of 1522 T1N0M0 DTC patients, Wang et al (13) found no difference in disease-specific survival among 1,522 patients with $\mathrm{T} 1$ tumors, and there was no difference in the risk of recurrence between total thyroidectomy and less than total 


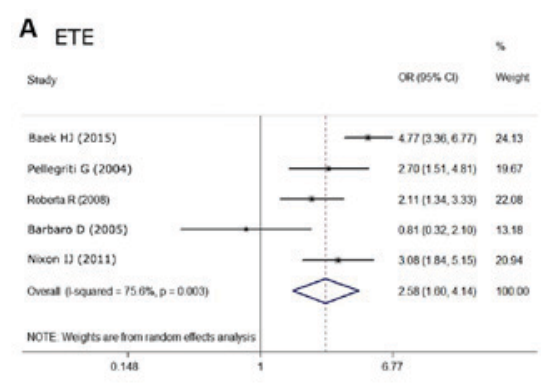

B Bilaterality

C Vascular invasion
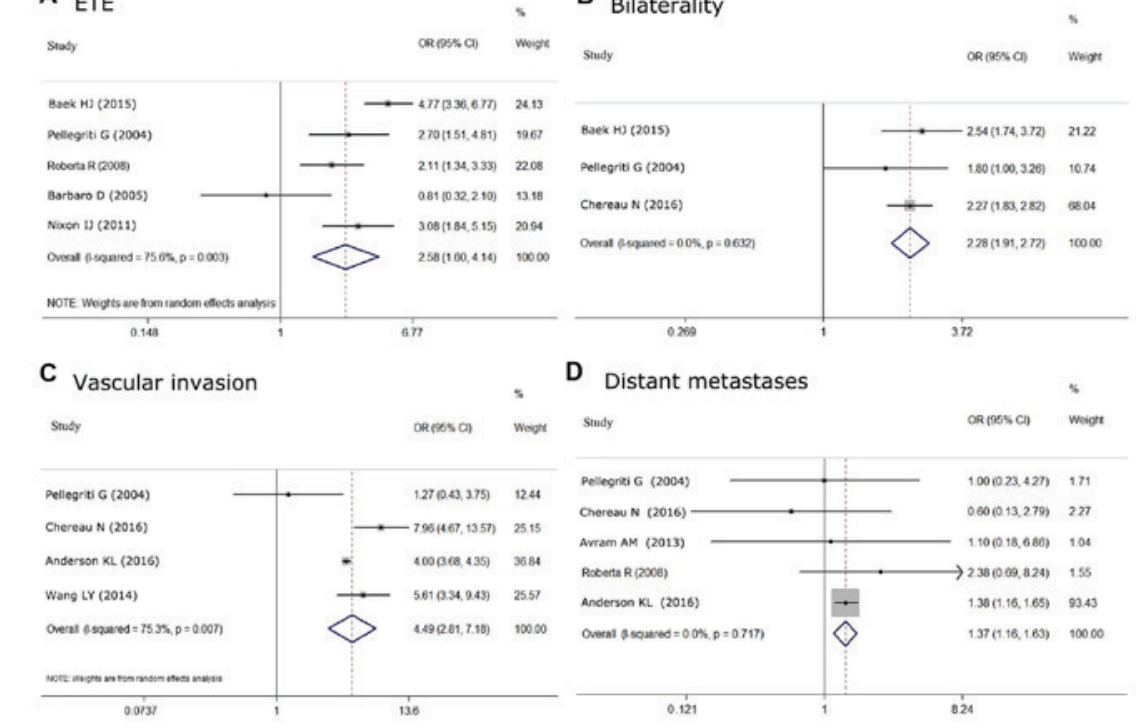

Distant metastases
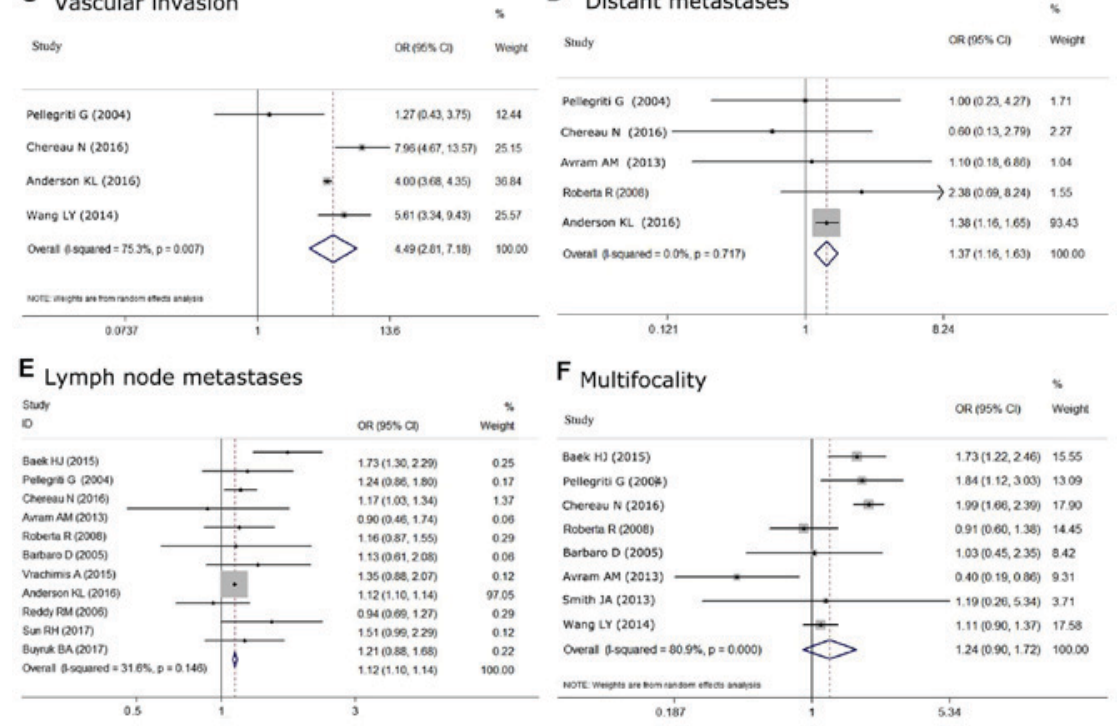

Figure 6. Associations with aggressive histologic features between subgroups (S2 vs. S1) are shown as the OR with 95\% CIs. (A) The random effects model for the association with ETE. (B) The fixed effects model for the correlation with bilaterality. (C) The random effects model for the correlation with vascular invasion. (D) The fixed effects model for the correlation with distant metastases. (E) The fixed effects model for the correlation with lymph node metastases. (F) The random effects model for the correlation with multifocality. CI, confidence interval; OR, odds ratio; sDTC, small-differentiated thyroid carcinoma; ETE, extrathyroidal extension.

thyroidectomy both for the patients with T1a and T1b (T1a: $\mathrm{P}=0.105$; T1b: $\mathrm{P}=0.868$ ).

The major limitation of this meta-analysis was that only 21 studies were suitable based on the inclusion and exclusion criteria. Although 358 articles were identified, most of the studies did not offer sufficient statistics about recurrence or survival subdivided among patients with $S 1$ and $S 2$ disease. There were few studies with the specific aim of investigating the effect of tumor size on patient prognosis. Thus, large-scale prospective studies are required to confirm the influence of tumor size on prognosis in SDTC. Moreover, since there were few data regarding the association between pathological type and subgroup (S1 and $S 2$ ), we could not adequately perform analyses on these factors. The limitation of insufficient availability of studies included resulted in some findings being heavily weighed by these few studies. Finally, publication bias is a major concern in all forms of pooled analyses, while our analysis revealed that this was not a complicating variable for any of the included studies.

Our meta-analysis suggested that patients with $S 2$ have an increased risk of postoperative recurrence and mortality compared with $S 1$ patients, and comparisons between T1a and T1b came to the same conclusions. Furthermore, the meta-analysis indicated that $S 2$ sDTC was more commonly associated with ETE, bilaterality, vascular invasion, lymph node metastases and distant metastases compared with $S 1$. In summary, our analysis suggests that it is necessary to subdivide sDTC into $S 1$ and $S 2$ subgroups due to their different effects on prognosis, especially recurrence. Future prospective studies are required to confirm the influence of tumor size on prognosis in sDTC.

\section{Acknowledgements}

Not applicable.

\section{Funding}

The present study was supported by funds from the National Natural Science Foundation of China (no. 81702649 to NQ, nos. 81572622 and 81272934 to QHJ.

\section{Availability of data and materials}

The analyzed datasets generated during the study are available from the corresponding author on reasonable request.

\section{Authors' contributions}

TTZ, CFL, NQ, RLS and QHJ were involved in the conception and design of the study. SSW, DZH and GHS were involved in the acquisition of the clinical data and reviewing. TTZ, NQ, YXZ and YW analyzed and interpreted the data. TTZ, NQ, 
RLS and QHJ wrote the manuscript. All authors have read and approved the manuscript.

\section{Ethics approval and consent to participate}

The present study was approved by the Human Ethics Committee/Institutional Review Board of Fudan University Shanghai Cancer Center.

\section{Patient consent for publication}

Not applicable.

\section{Competing interests}

The authors declare that they have no competing interests.

\section{References}

1. Hedinger C, Williams E, and Sobin L: Histological typing of thyroid tumour. 2nd ed. World Health Organization, New York, NY, pp 15-17, 1993

2. Jemal A, Bray F, Center MM, Ferlay J, Ward E and Forman D: Global cancer statistics. CA Cancer J Clin 61: 69-90, 2011.

3. Chen AY, Jemal A and Ward EM: Increasing incidence of differentiated thyroid cancer in the United States, 1988-2005. Cancer 115: 3801-3807, 2009.

4. Lim H, Devesa SS, Sosa JA, Check D and Kitahara CM: Trends in thyroid cancer incidence and mortality in the United States, 1974-2013. JAMA 317: 1338-1348, 2017.

5. Nilubol N and Kebebew E: Should small papillary thyroid cancer be observed? A population-based study. Cancer 121: 1017-1024, 2015.

6. Vrachimis A, Wenning C, Gerß J, Dralle H, Vaez Tabassi M, Schober O, Riemann B and MSDS study group: Not all DTC patients with $\mathrm{N}$ positive disease deserve the attribution 'high risk'. Contribution of the MSDS trial. J Surg Oncol 112: 9-14, 2015.

7. Anderson KL Jr, Youngwirth LM, Scheri RP, Stang MT, Roman SA and Sosa JA: T1a versus T1b differentiated thyroid cancers: Do we need to make the distinction? Thyroid 26: 1046-1052, 2016

8. Bilimoria KY, Bentrem DJ, Ko CY, Stewart AK, Winchester DP, Talamonti MS and Sturgeon C: Extent of surgery affects survival for papillary thyroid cancer. Ann Surg 246: 375-381; discussion 381-374, 2007.

9. Edge SB and Compton CC: The american joint committee on cancer: The 7th edition of the AJCC cancer staging manual and the future of TNM. Ann Surg Oncol 17: 1471-1474, 2010.

10. Amin MB, Edge S, Greene F, Byrd DR, Brookland RK, Washington MK, Gershenwald JE, Compton CC, Hess KR, Sullivan DC, et al (eds): AJCC Cancer Staging Manual. Eighth edition. Springer, New York, NY, pp 873-887, 2017.

11. Haugen BR, Alexander EK, Bible KC, Doherty GM, Mandel SJ, Nikiforov YE, Pacini F, Randolph GW, Sawka AM, Schlumberger M, et al: 2015 American thyroid association management guidelines for adult patients with thyroid nodules and differentiated thyroid cancer: The american thyroid association guidelines task force on thyroid nodules and differentiated thyroid cancer. Thyroid 26: 1-133, 2016.

12. Chereau N, Tresallet C, Noullet S, Godiris-Petit G, Tissier F, Leenhardt L and Menegaux F: Does the T1 subdivision correlate with the risk of recurrence of papillary thyroid cancer? Langenbecks Arch Surg 401: 223-230, 2016.

13. Wang LY, Nixon IJ, Palmer FL, Thomas D, Tuttle RM, Shaha AR, Patel SG, Shah JP and Ganly I: Comparable outcomes for patients with pT1a and pT1b differentiated thyroid cancer: Is there a need for change in the AJCC classification system? Surgery 156: $1484-1490,2014$
14. Rossi R, Roti E, Trasforini G, Pansini G, Cavazzini L, Zatelli MC, Pearce EN, Braverman LE and Uberti EC: Differentiated thyroid cancers $11-20 \mathrm{~mm}$ in diameter have clinical and histopathologic characteristics suggesting higher aggressiveness than those $\leq 10 \mathrm{~mm}$. Thyroid 18: 309-315, 2008 .

15. Moher D, Shamseer L, Clarke M, Ghersi D, Liberati A, Petticrew M, Shekelle P, Stewart LA and PRISMA-PGroup: Preferred reporting items for systematic review and meta-analysis protocols (PRISMA-P) 2015 statement. Syst Rev 4: 1, 2015.

16. Stang A: Critical evaluation of the Newcastle-Ottawa scale for the assessment of the quality of nonrandomized studies in meta-analyses. Eur J Epidemiol 25: 603-605, 2010.

17. Ito $\mathrm{Y}$, Masuoka $\mathrm{H}$, Fukushima $M$, Inoue $\mathrm{H}$, Kihara $M$, Tomoda C, Higashiyama T, Takamura Y, Kobayashi K, Miya A and Miyauchi A: Excellent prognosis of patients with solitary T1N0M0 papillary thyroid carcinoma who underwent thyroidectomy and elective lymph node dissection without radioiodine therapy. World J Surg 34: 1285-1290, 2010.

18. Pellegriti G, Scollo C, Lumera G, Regalbuto C, Vigneri R and Belfiore A: Clinical behavior and outcome of papillary thyroid cancers smaller than $1.5 \mathrm{~cm}$ in diameter: Study of 299 cases. J Clin Endocrinol Metab 89: 3713-3720, 2004.

19. Barbaro D, Simi U, Meucci G, Lapi P, Orsini P and Pasquini C: Thyroid papillary cancers: Microcarcinoma and carcinoma, incidental cancers and non-incidental cancers-are they different diseases? Clin Endocrinol (Oxf) 63: 577-581, 2005

20. Reddy RM, Grigsby PW, Moley JF and Hall BL: Lymph node metastases in differentiated thyroid cancer under $2 \mathrm{~cm}$. Surgery 140: 1050-1054; discussion 1054-1055, 2006.

21. Krämer JA, Schmid KW, Dralle H, Dietlein M, Schicha H, Lerch H, Gerss J, Frankewitsch T, Schober O, Riemann B and MSDS study group: Primary tumour size is a prognostic parameter in patients suffering from differentiated thyroid carcinoma with extrathyroidal growth: Results of the MSDS trial. Eur J Endocrinol 163: 637-644, 2010.

22. Nixon IJ, Ganly I, Patel S, Palmer FL, Whitcher MM, Tuttle RM, Shaha AR and Shah JP: The impact of microscopic extrathyroid extension on outcome in patients with clinical T1 and T2 well-differentiated thyroid cancer. Surgery 150: 1242-1249, 2011.

23. Ibrahimpasic T, Nixon IJ, Palmer FL, Whitcher MM, Tuttle RM, Shaha A, Patel SG, Shah JP and Ganly I: Undetectable thyroglobulin after total thyroidectomy in patients with low- and intermediate-risk papillary thyroid cancer-is there a need for radioactive iodine therapy? Surgery 152: 1096-1105, 2012

24. Avram AM, Fig LM, Frey KA, Gross MD and Wong KK: Preablation 131-I scans with SPECT/CT in postoperative thyroid cancer patients: What is the impact on staging? J Clin Endocrinol Metab 98: 1163-1171, 2013.

25. Smith JA, Sharma N, Nankivell P and Watkinson JC: The presentation, natural history and outcome of $\mathrm{T} 1 \mathrm{a} / \mathrm{b}$ thyroid cancer with regard to new grading systems. Eur Arch Otorhinolaryngol 272: 439-444, 2015.

26. Momesso DP, Vaisman F, Caminha LS, Pessoa CH, Corbo R and Vaisman M: Surgical approach and radioactive iodine therapy for small well-differentiated thyroid cancer. J Endocrinol Invest 37: 57-64, 2014.

27. Han JM, Kim WG, Kim TY, Jeon MJ, Ryu JS, Song DE, Hong SJ, Shong YK and Kim WB: Effects of low-dose and high-dose postoperative radioiodine therapy on the clinical outcome in patients with small differentiated thyroid cancer having microscopic extrathyroidal extension. Thyroid 24: 820-825, 2014.

28. Baek HJ, Kim DW and Ryu JH: Association between TNM staging system and histopathological features in patients with papillary thyroid carcinoma. Endocrine 48: 589-594, 2015.

29. Sun R, Zhang H, Liu K, Fan J, Li G, Song X and Li C: Clinicopathologic predictive factors of cervical lymph node metastasis in differentiated thyroid cancer. Acta Otorrinolaringol Esp 69: 149-155, 2018 (In English, Spanish).

30. Aydin Buyruk B, Kebapci N, Yorulmaz G, Buyruk A and Kebapci M: An evaluation of clinicopathological factors effective in the development of central and lateral lymph node metastasis in papillary thyroid cancer. J Natl Med Assoc 110: 384-390, 2018.

This work is licensed under a Creative Common Attribution 4.0 International (CC BY 4.0) License. 